\title{
Analysis and optimization of cross-rail structure of milling composite extrusion CNC machine
}

\author{
Zhifeng Liu, Zheng Shi, Tieneng Guo \\ Beijing University of Technology, Beijing 100124, China \\ Zhijie Li \\ North China Institute of Aerospace Engineering, Langfang 065000, China
}

\begin{abstract}
Cross-rail is a key component of support structure of the machine, whose mechanical properties have direct impact on the accuracy and dynamic performance of the machine. This paper studied a bridge type milling composite extrusion CNC machine whose cross-rail welded together by a rib structure, therefore, well-designed rib structure is particularly necessary. In this paper, the finite element method for analyzing the cross-rails of four different rib structures, static deformation and natural frequency, and the deformation of impact on the process of start and stop in three performances are adopted to obtain better overall performance rib structure to meet the requirements of the mechanical properties of the machine.
\end{abstract}

KEYWORD: Machine cross-rail, Finite element, Ribs, Static and dynamic characteristics

\section{INTRODUCTION}

Milling composite extrusion CNC machine makes multiple processes, such as high-speed milling process and progressive extrusion molding, etc. integrated on a single device with high efficiency, composite, heavy-duty and high precision machining. It is mainly used in aerospace protective sheet metal processing, which can greatly enhance the processing efficiency and quality of our spacecraft. Cross-rail is a key component of support structure of the machine, whose mechanical properties have direct impact on the accuracy and dynamic performance of the machine.

In terms of domestic and foreign research of machine tool, finite element method is mainly used for the assessment of structural static, dynamic rigidity and dynamic stability[1,2]. Xiaoyu Wang [3] proposed a topology optimization method for longitudinal and cross-section of cross-rail of gantry machining center in the form of ribs distributed. Hongling Hou [4] analysed several different crosssectional shape of the structure of cross-rail for static and modal, getting a much better stiffness of crossrail from the better intensity of cross-rails by using modal analysis. Yanqing Wang [5] studied four structures of large five-axis machining center, comparing the maximum deformation by using static analysis and selected the best structure. This paper studied the cross-rail of bridge type milling composite extrusion CNC machine. Finite element method is used to analyze the static and dynamic characteris- tics of the cross-rail, and finally determine the best structure by comparing several different forms of structure.

\section{RIB DESIGN OF CROSS-RAIL}

\subsection{The cross-rail profile:}

Cross-rail in this study is about 8.2meters, and the quality is 10 tons, as shown in figure 1-1.The global coordinate system is as follows. Anti-gravity is the $\mathrm{Z}$ direction, $\mathrm{X}$ is the longitudinal direction, front and rear direction is $\mathrm{Y}$ direction.

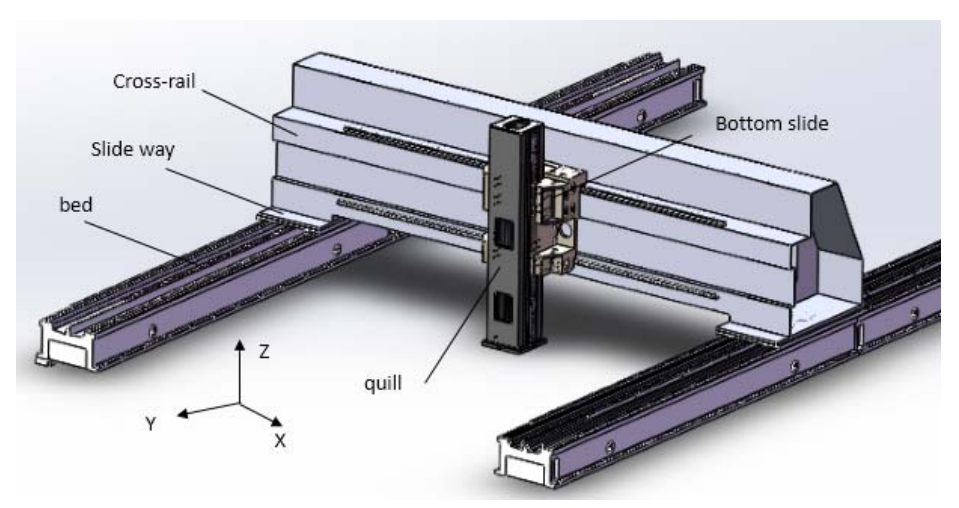

Fig. 1-1 milling composite extrusion CNC machine and global coordinate

The cross-rail material is Q235. The property of the cross-rail material is as showing in table 1-1. 
Table 1-1 Cross-rail material parameters

\begin{tabular}{llll}
\hline Material & $\begin{array}{l}\text { Density } \\
\left(\mathrm{kg} / \mathrm{m}^{3}\right)\end{array}$ & $\begin{array}{l}\text { Young's modulus } \\
(\mathrm{GP})\end{array}$ & $\begin{array}{l}\text { Poisson's ra- } \\
\text { tio }\end{array}$ \\
\hline Q235 & 7850 & 200 & 0.3 \\
\hline
\end{tabular}

\subsection{The Rib design:}

The force of cross-rail of bridge type machining center is formed as a simply supported beam supported at both ends of the beam. In addition, it bears the force caused by the weight of cross-rail itself, ram, slide, spindle, etc but also bears the overturning and torsional torque caused by the spindle box and etc. Different rib structures have different bending and torsional capabilities. The effect of rib is transmitting local load to the others so that the entire support member can withstand relatively uniform load. Longitudinal partitions can improve the bending stiffness, transverse partition can improve torsional rigidity and not only can oblique separator improve bending stiffness, but also improve the torsional stiffens. Therefore, choosing the right arrangement of rib structure is necessary to increase the bending and torsion capability of cross-rail. This paper conducted three types of improvements based on the initial rib structure of cross-rail, analyzed respectively static deformation of cross-rail assembly with slide plate and the ram and the deformation of cross-rail with horizontal acceleration of $0.5 \mathrm{~g}$. Through several different forms of comparative analysis of the structures ultimately the best solution is determined. Several rib structures of the cross-rail are as follows:

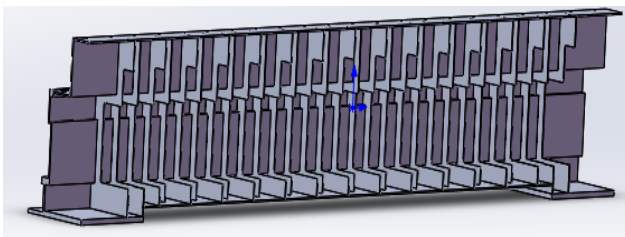

(a)

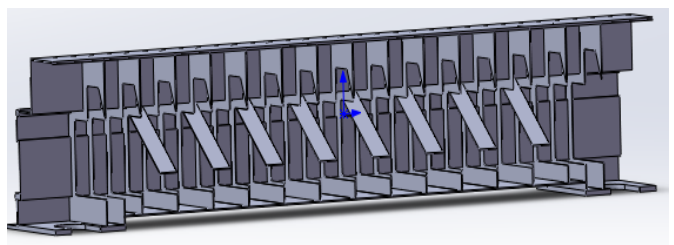

(b)

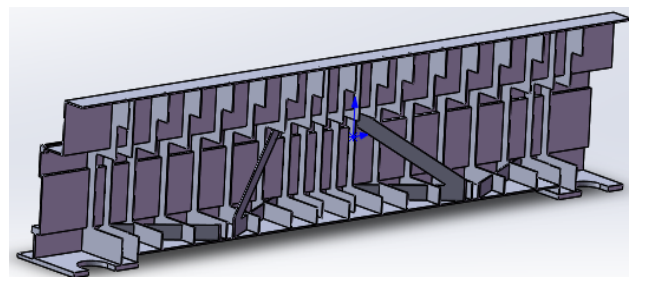

(c)

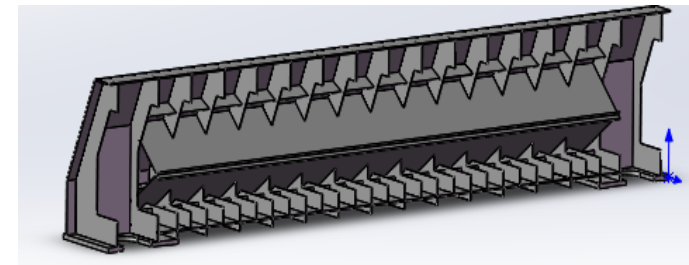

(d)

Fig. 1-2 Four kinds of rib structures of cross-rail (isometric)

The rib thickness of cross-rail (a), (b), (d) is $10 \mathrm{~mm}$, The rib thickness of both ends of cross-rail (c) is $10 \mathrm{~mm}$, the middle rib thickness is $15 \mathrm{~mm}$. Consider diagonal ribs can enhance the ability to torsional bending, cross-rail (b) increase eight diagonal ribs based on the cross-rail (a), the bottom of cross-rail (c) increase two crossed oblique ribs, intermediate of the cross-rail (c) increase two oblique ribs, as shown in Figure 1-3 (c).

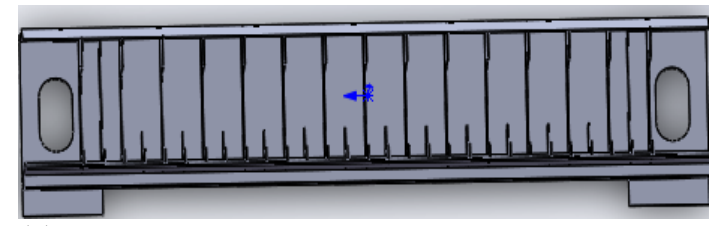

(a)

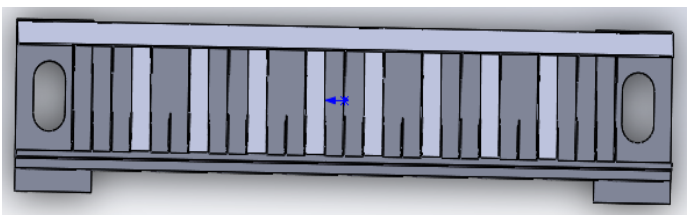

(b)

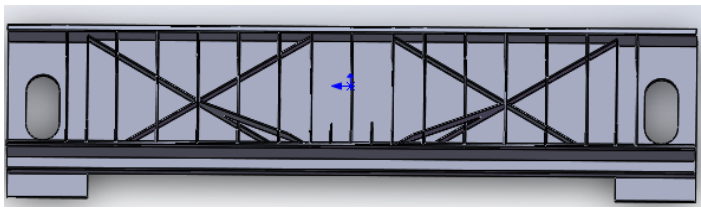

(c)

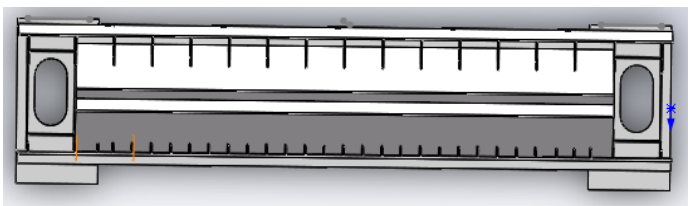

(d)

Fig. 1-3 Four kinds of rib structures of cross-rail (top)

\section{STATIC ANALYSIS OF CROSS-RAIL:}

The CAD model is imported into finite element analysis software of ANSYS workbench. Material of cross-rail is steel Q235, Material of sliding plate and ram is gray cast iron HT300, Material parameters: elastic modulus: $126 \mathrm{GPa}$, Poisson's ratio: 0.27 , density: $7200 \mathrm{~kg} / \mathrm{m}^{3}$. Using the free grid method, the cell size $40 \mathrm{~mm}$, using the solid186 unit for grid division. The surface of both ends of the cross-rail contacted 
with the carriage is constrained to the vertical $\mathrm{Z}$, as shown in Figure 2-1 (a). The cylindrical coordinates is applied to the position of the motor at one end of the cross-rail, horizontal $\mathrm{Y}$ direction which is $\mathrm{X}$ direction of cylindrical coordinates is constrained, as shown in Figure 2-1 (b). The other end of the crossrail as the symmetry position only horizontal Y direction is constrained, as shown in Figure 2-1 (c). The three constraint are formed to simulate the real working conditions of cross-rail to get more accurate solution.

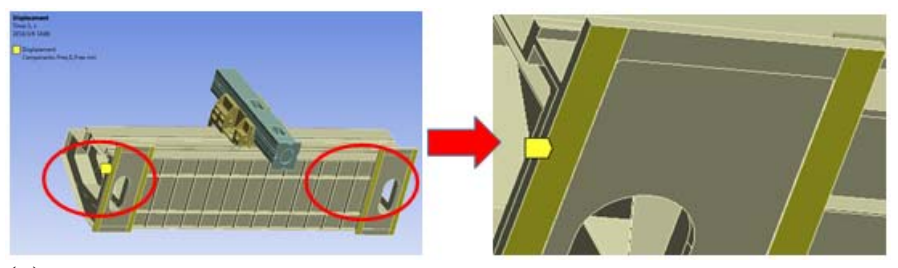

(a)

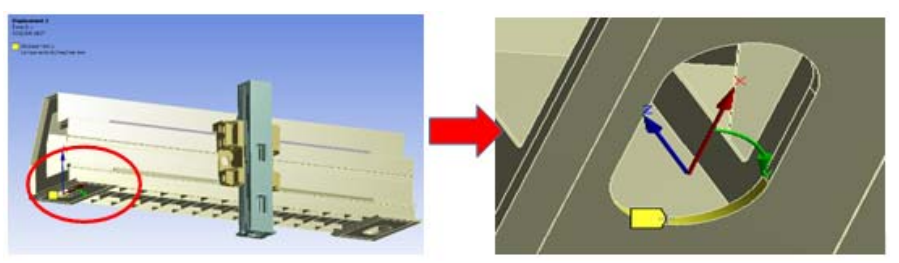

(b)

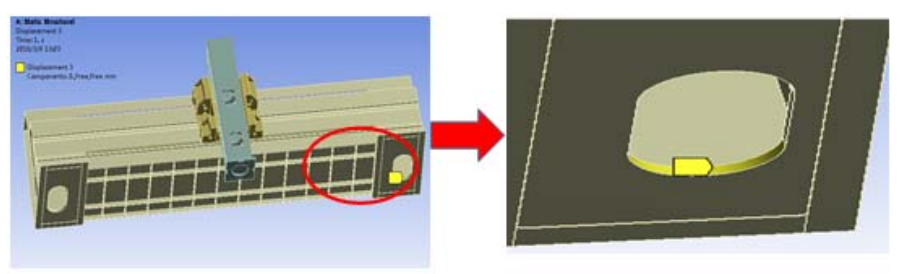

(c)

Fig. 2-1 Forms in the constraints of cross-rail

Calculating the static deformation of the cross-rail in the limit condition assembly with ram and the slide plate (considering the weight of the other attached parts added to the slide and the ram, increased to a total of about 9 tons). Ram and the slide plate are in the middle position of the cross-rail, the limit of the vertical force of the cross-rail is simulated, and the maximum deformation of the vertical $\mathrm{Z}$ is analyzed, as shown in figure 2-2.

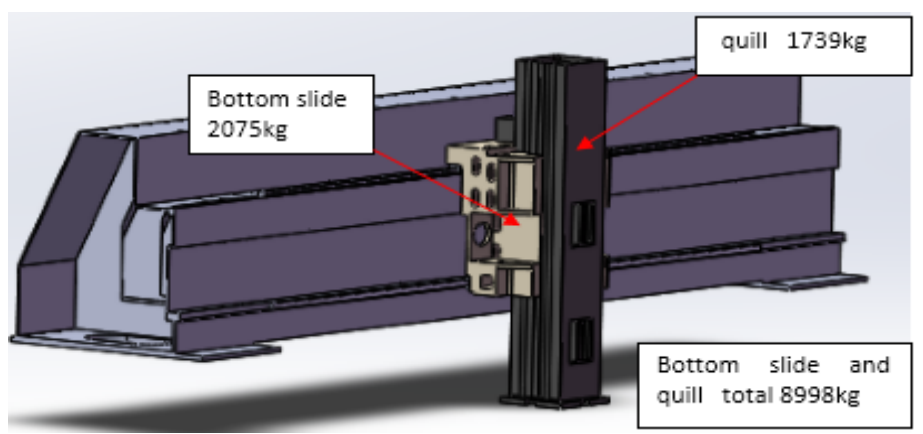

Fig. 2-2 Cross-rail assembly
Through calculation and analysis, the deformation of cross-rail (a) is maximum, vertical maximum deformation is about $0.28 \mathrm{~mm}$. The vertical maximum deformation of cross-rail (b) is about $0.28 \mathrm{~mm}$, but the area of maximum deformation is less than the cross-rail (a), the maximum deformation of crossrail (c) is $0.23 \mathrm{~mm}$ around, the vertical maximum deformation of cross-rail (d) is minimum which is 0.19 $\mathrm{mm}$ around, it is found that the cross-rail (d) has more obvious effect of resistance static deformation by comparing above, the maximum deformation polyline of four kinds of structure form is shown in figure 2-3.

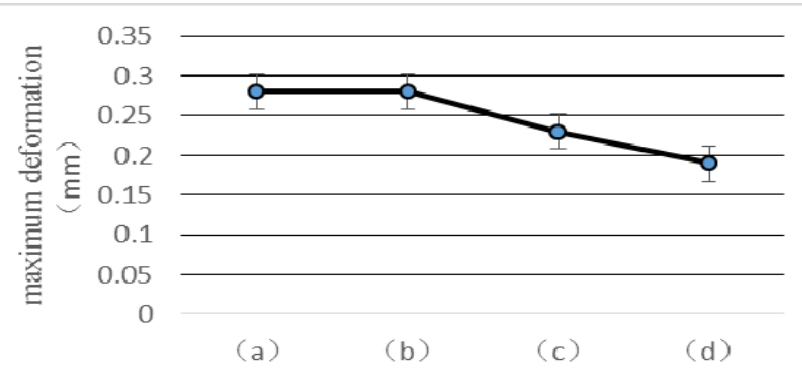

Fig. 2-3 Comparison of four kinds of cross-rail deformation

\section{DYNAMIC ANALYSIS OF CROSS-RAIL:}

\subsection{Modal analysis of cross-rail:}

It is not enough to analyze the static characteristic of the four rib structures of the cross-rail, so it is necessary to analyze the dynamic characteristics of the four kinds of rib structure. The several lower natural frequencies of the component have the greatest impact on its performance, therefore, the first five modes of the cross-rails of the four rib structures are studied. The first to the fifth order of the natural frequency list of four rib structures is as shown in figure 3-1, the frequency comparison chart of the polyline as shown in figure 3-2. It can be seen from the figure that the first two frequencies of the four structures are not quite different. In addition to the second stage, the natural frequencies of the cross-rail (d) are significantly higher than those of the other three cross-rails. So the vibration resistance of cross stiffened plate structure of cross-rail (d) is better than that of other three kinds of beams, and the first order vibration mode of the four rib structures of the cross-rail is shown in figure 3-2.

\begin{tabular}{l|llll}
\hline Natural frequency $(\mathrm{Hz})$ & (a) & (b) & (c) & (d) \\
Order & & & & \\
\hline 1 & 92 & 94 & 95 & 104 \\
2 & 113 & 116 & 117 & 114 \\
3 & 116 & 124 & 123 & 202 \\
4 & 122 & 169 & 181 & 209 \\
5 & 133 & 185 & 192 & 225 \\
\hline
\end{tabular}

Fig. 3-1 The first five natural frequencies of cross-rails 


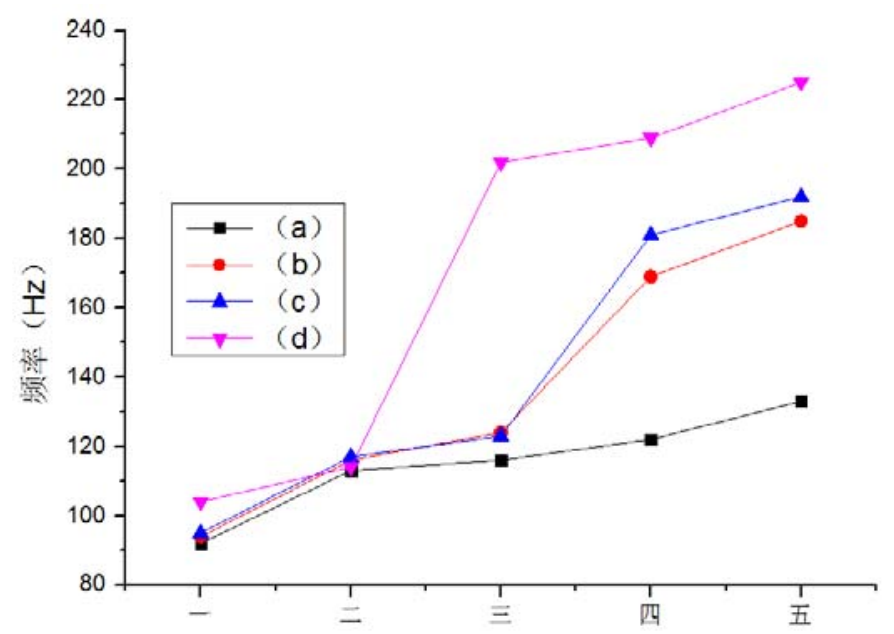

Fig. 3-2 Comparison of the first five natural frequencies of cross-rails

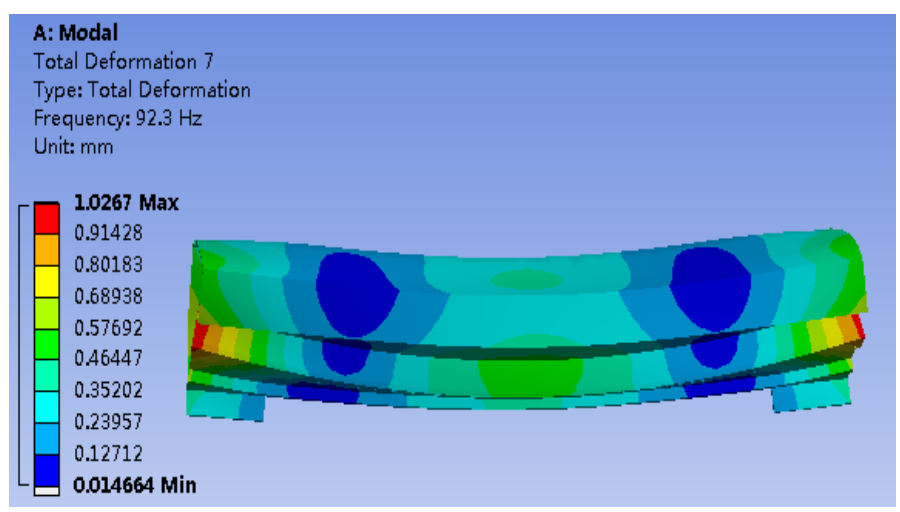

(a)

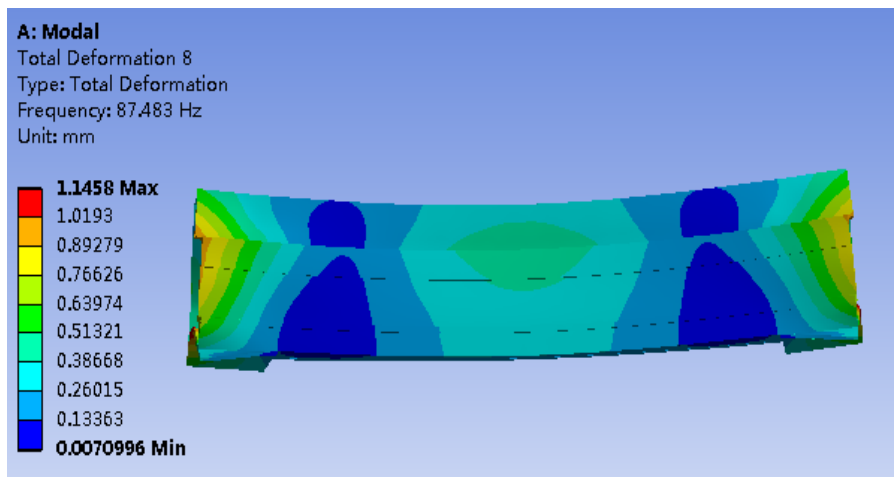

(b)

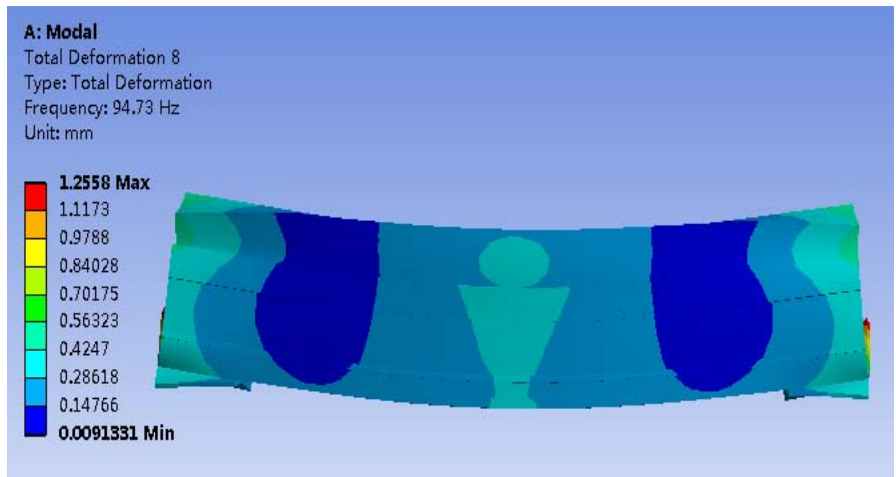

(c)

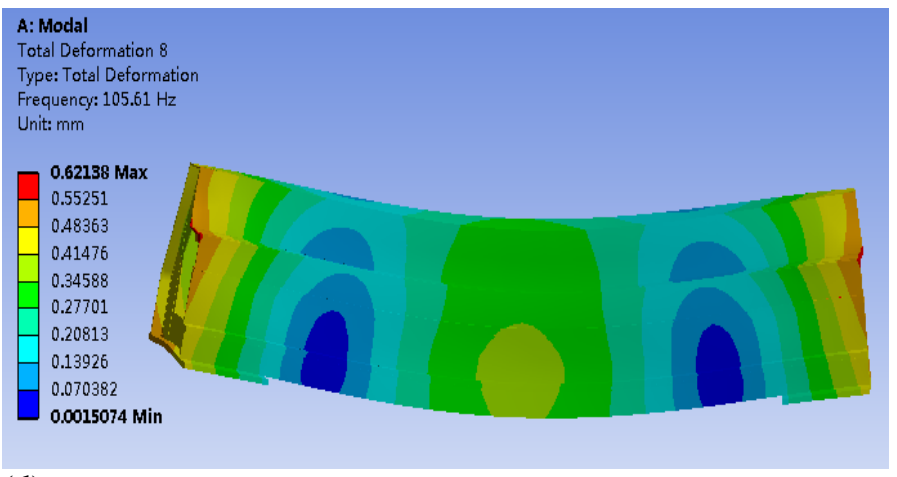

(d)

Fig. 3-3 The first order modal shape of four cross-rails

\subsection{Deformation of horizontal $0.5 \mathrm{~g}$ acceleration:}

Without taking into account the weight of cross-rail, the analysis of the steady state deformation under the condition of constant acceleration of $0.5 \mathrm{~g}$ can be obtained by the direct action of a $0.5 \mathrm{~g}$ constant acceleration force field in the transverse direction. During startup by $0.5 \mathrm{~g}$ acceleration (acceleration suddenly change from zero to $0.5 \mathrm{~g}$ ), the dynamic deformation will not exceed the steady-state deformation of $0.5 \mathrm{~g}$ acceleration. The conclusion can be understood as the deformation of cross-rail produced by $0.5 \mathrm{~g}$ acceleration is the equilibrium position, and the deformation amount is initial deformation away from the equilibrium position, the peak value of the vibration displacement around the equilibrium position is greater than the initial displacement value.

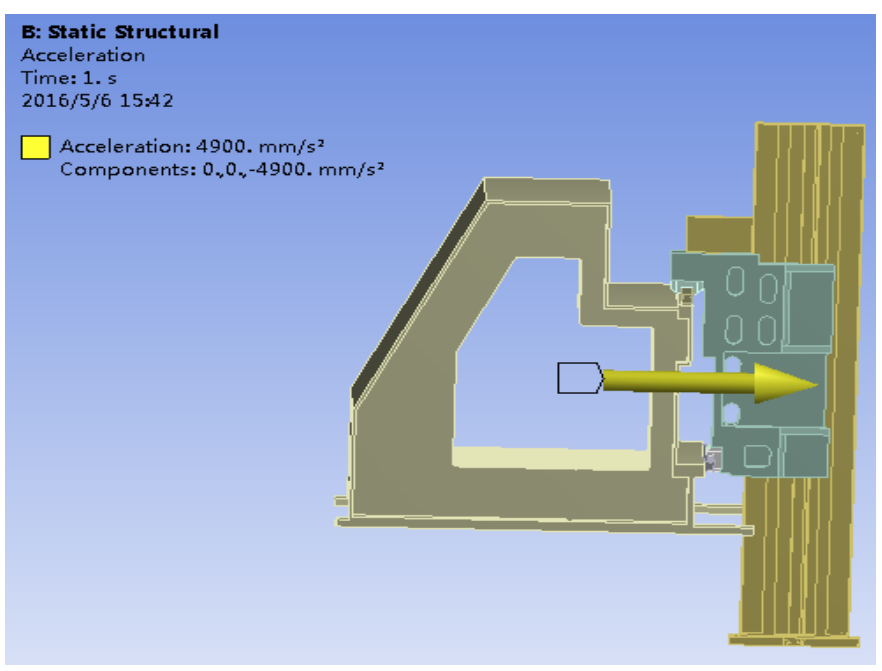

Fig. 3-4 0.5g acceleration of horizontal y

Through the calculation and analysis the horizontal y maximum deformation of cross-rail (a) is about $0.24 \mathrm{~mm}$, the maximum deformation of cross-rail (b) is $0.21 \mathrm{~mm}$ around, the maximum deformation of cross rail (c) and (b) have little difference and is about $0.22 \mathrm{~mm}$. Deformation of the cross-rail (d) is minimum, the maximum deformation of which is about $0.14 \mathrm{~mm}$. By comparing the analysis we know the dynamic characteristics of the cross-rail (d) is 
higher than the others, and its technological requirements is not high, it is easy to manufacture, as shown in figure 3-4.

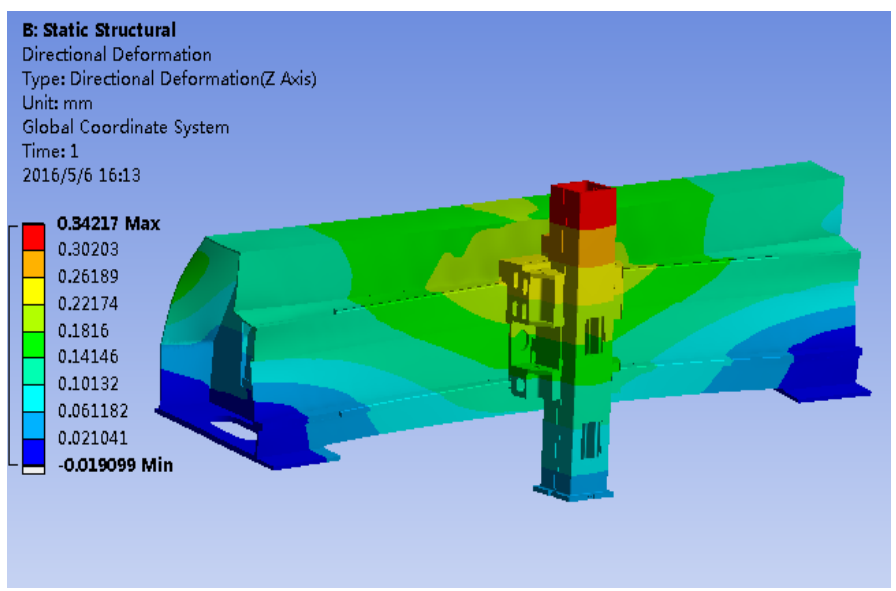

(a)

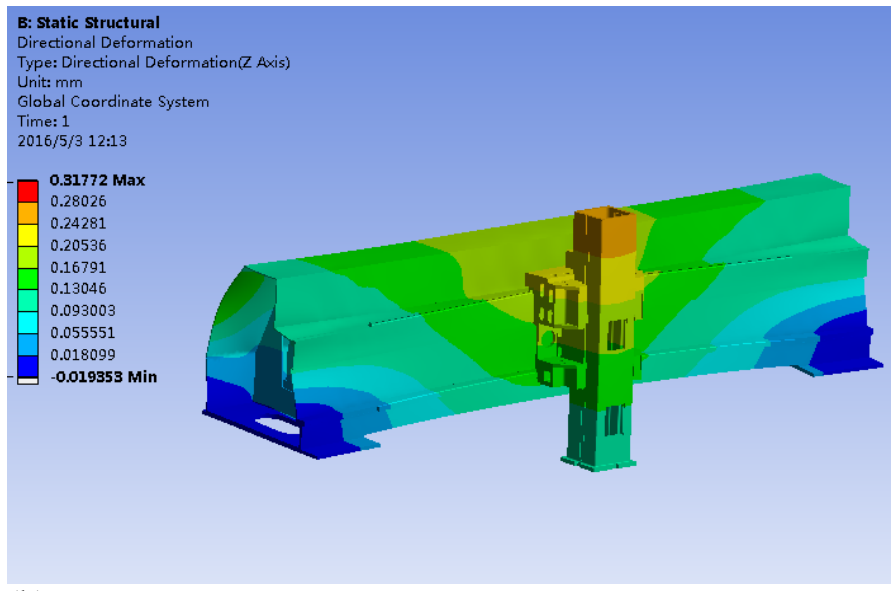

(b)

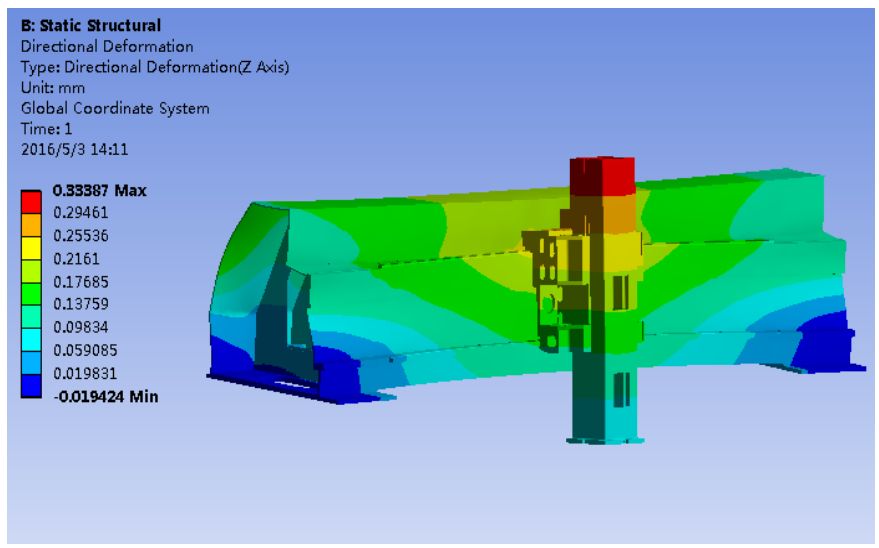

(c)
A: Static Structural

Type: Directional Deformation $\alpha$ Axis)

Unit: $\mathrm{mm}$

Global Coordinate System

Time: 1

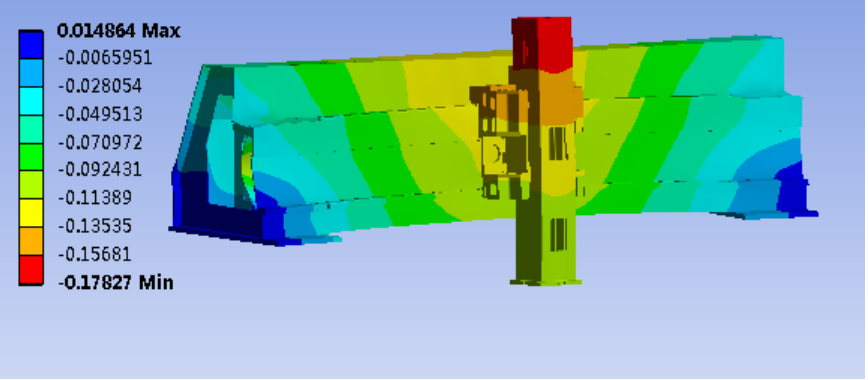

(d)

Fig. 3-5 Horizontal deformation of the cross-rails

\section{SUMMARY}

Based on the above analysis, the following conclusions can be drawn: 1. Among four types of rib structure of cross-rail, the ability to resist deformation of cross-rail (a) is the worst, the vertical maximum static deformation is 1.47 times than the cross-rail (d), bending and torsional resistance of cross stiffened rib structure of cross-rail (d) is better than the other three structures. 2 .Though the thickness of the rib of cross-rail (c) is $5 \mathrm{~mm}$ larger than cross-rail (a), the performance of overall stiffness has not been significantly improved. 3. The natural frequencies of cross-rail (d) are basically higher than those of the other three structures and the natural frequencies after the third order are significantly higher than other three. (the third-order is $100 \mathrm{~Hz}$ higher than cross-rail (a), $78 \mathrm{~Hz}$ higher than crossrail (b), $79 \mathrm{~Hz}$ higher than cross-rail(c)), under $0.5 \mathrm{~g}$ acceleration the maximum deformation of horizontal $y$ of the cross-rail (a) is 1.71 times than cross-rail (d), so the dynamic characteristics of cross-rail (d) is much better than the other three.

\section{REFERENCES}

[1] BIANCH IG, PAOLUCC IF. Towards virtual engineering in machine tool design[J]. CIRP Annals Manufacturing Technology, 1996, 45(1):619-621.

[2] ChuanLin Luo, DuanNeng Li.Design and research of the structural of beam in the planer type machines [J]. Mechanical \& Electrical Engineering, 2006(3):45-47.

[3] XiaoYu Wang, ZhenYuan Jia, Fan Yang. The topological optimization design and analysis for gantry machine tool crossbeam component[J].Manufacturing Technology \& Machine Tool, 2009, (11): 64-68.

[4] Hong Ling Hou, Zhi Hui Qiu, Yong Qiang Zhao. Static \& dynamic analysis for beam of high -speed cutting machine tool [J]. Machinery Design \& Manufacture, 2006(5):38-39.

[5] YanQing Wang, GaoYan Zhong, YongBiao Chang. Design of beam structures for large five - axis machining center [J]. Machine Tool \& Hydraulics, 2012, 40(13):114-117. 